\title{
Early Delirium Assessment for Hospitalized Older People in Indonesia: A Systematic Review
}

\author{
Azam David Saifullah ${ }^{1}$, Ching-Min Chen ${ }^{2}$ \\ ${ }^{1,2}$ National Cheng Kung University, Taiwan \\ Corresponding author: azamsaifullah@gmail.com
}

\begin{abstract}
Background: Due to the increasing risk of getting co-morbidity and frailty, older people tend to be prone to hospitalization. Hospitalization in older people brings many adverse effects. Moreover, when these elderly get delirium, the mortality and morbidity will increase. The risk of getting deterioration and worsening condition because of delirium would also increase. In fact, delirium assessment is not a high priority in taking care older people during hospitalization because the focus of care is treating the disease. Delirium screening as an early recognition of delirium in the hospitalized elderly in Indonesia remains unreported and even do not well evaluated. Therefore, delirium as a preventable problem or causing problems remains unrecognized.

Purpose: This paper aims to review the current evidence of early assessment of delirium in hospitalized older people.

Methods: A systematic review was conducted from four databases yielding to 4 articles which met the inclusion and exclusion criteria.

Results: There are four focuses on the result, namely delirium screening tools, patient characteristics, identified early delirium assessment, and outcomes affected by early delirium assessment. Confusion Assessment Method (CAM) was used as the delirium screening tool in the hospital. Establishing the care team involving many disciplines will give a better way to improve the integrated care and collaborative care.

Conclusion: Performing CAM integrated in comprehensive geriatric assessment can be the most important thing to be undertaken when looking after the hospitalized elderly.
\end{abstract}

Keywords: delirium, assessment, older people 


\section{BACKGROUND}

The majority of patients in hospitals and community are older people (Tadd et al., 2011). Complication of chronic diseases is a major cause of hospital re-admission (World Population Ageing, 2013) and even disability among older people (Gill, Allore, Holford, \& Guo, 2004). The vulnerability of the older people caused by aging and chronic disease makes older people become frail (Nagamine, Jiang, \& Merrill, 2006). Furthermore, frailty among older people take $26 \%$ of hospitalization, chronic disease and geriatric syndrome increase the risk of hospitalization among older people (Fagerberg \& Engström, 2012).

During hospitalization, older people will receive treatments and interventions depending on the complaint or disease. Researches show that during hospitalization, older people increase their risk to develop functional symptoms of adverse effect by $5.7 \%$. The functional symptoms of adverse effect are confusion, less appetite, and incontinence. Older people receive treatment and intervention in consequence of the functional symptoms (Gillick, Serrell, \& Gillick, 1982; Nagamine et al., 2006).

In general, the adverse effect of hospitalization in older people will increase the risk of mortality and prolong the hospital stay. Prolonged hospital stays for older people means that they will have less physical activity in hospital compared to the activity at home which can affect their functional ability (Isfandiaty, 2012; Schuurmans, Duursma, \& Shortridge-Baggett, 2001). In relation to hospitalization in older people, age was associated with the failure to recover ADL function during hospitalization in patients who declined before admission (OR for patients aged 90 compared with patients aged 70-74=2.09, 95\% CI=1.20-3.65) and with new losses of ADL function during hospitalization in patients who did not decline before admission (OR for patients aged 90 compared with patients aged 70-74=3.43, 95\% CI=1.92-6.12) (Covinsky et al., 2003).

In those hospitalization adverse effects, older people need both physical and psychological interventions. Some medical interventions, which are usually delivered to reduce functional symptoms directly, are psychotropic medication, restraints, nasogastric tube, and foley catheter. However, these medical interventions intended to reduce functional symptoms lead to a depressed psycho-physiological functioning (Gillick et al., 1982). One of the most common problems is delirium. Compared to other developing countries, in Indonesia, the percentage of hospitalized elderly patients at the inpatient units of Cipto Mangunkusumo Hospital, Jakarta (RSCM) in 2001 and 2002 was as high as 26.3 and $28.7 \%$, respectively. Delirium, or acute confusion state, is a common diagnosis with serious complications in hospitalized elderly patients (Isfandiaty, 2012).

The prevalence of delirium on admission varied from 14 to 56\% with associated hospital mortality rate of $25-30 \%$. In Acute Geriatric Ward of RSCM, the incidence of delirium was around $17 \%$ with a prevalence of up to $47.3 \%$ (Isfandiaty, 2012). Delirium can be simply defined as a mental disorder presented with global cognitive dysfunction, alter of consciousness, sleep cycle and psychomotor activity in the acute setting and fluctuated. 
Delirious patients have increased morbidity, decreased functional status, prolonged hospital stay and increased risk of entering long-term care on discharge from hospital compared to the patients without delirium (Rigney, 2006; Schuurmans et al., 2001). A systematic literature study showed the mean of hospitalization and associated mortality rates after 6 months period were $36 \%$ and 26\%, respectively. Delirium occurs as a consequence of interrelation of predisposing factors (risk factors) and precipitating factors (triggering factors). In general, the risk factors are age, dementia, hypoalbuminemia, usage of anticholinergic, and psychoactive medications. Delirium usually happens in the early hospitalization which means that it can be prevented (Hsieh, Ely, \& Gong, 2013). The current delirium care suggested involving one important part which is the assessment of delirium in the early stage of admission to the ward. Early screening, or recognition of delirium can increase the delirium prevention program for certain patients (Schuurmans et al., 2001).

In papers, there is still limited hospital that provides older people care in terms of hospital care. There are nine hospitals, which has integrated clinic for older people, and only four of them have geriatric ward $(\mathrm{MoH}, 2014)$. Resources in each ward usually consist of many medical providers such as nurse, physician, geriatric specialist, pharmacist, and dietician (Health, 2015). The nurses are divided into two roles in nursing model in Indonesia which are primary nurse and associate nurse. Primary nurse, together with the physician will have the function of most assessment of the patient (Rosanti, 2006). The assessment and the care planning are done in collaborative way, mostly promoted in the geriatric ward. However, the development of care in the ward mostly focuses on how to deliver the care in treating the disease, while delirium care becomes less priority. In one cohort study, it was found that some data in several variables are missing due to incompleteness of medical records (Isfandiaty, 2012).

Moreover, the diagnosis of delirium is frequently missed by doctors or other hospital team, either because the diagnosis of delirium is difficult to establish or because low awareness of those who have a high risk for development of delirium. In a study, staffs recognized less than $21 \%$ of patients with delirium, $33 \%$ of patients with dementia, and $36 \%$ of cognitively impaired patients. In facts, this shows that delirium is less recognized by the staffs (Wand et al., 2013). There is no difference in outcomes between English and non-English speaking patients. However, there is not yet a review on effect of early delirium assessment whether it improves the health care outcomes. Therefore, it is a need to provide what are the outcomes of early delirium assessment for hospitalized older people through evidence based practice. Therefore, this study aimed to assess current available literature in early delirium assessment outcomes in hospitalized older people.

\section{OBJECTIVE}

This paper aims to review the current evidence of early assessment of delirium in hospitalized older people.

\section{METHODS}

The study was conducted in systematic review to obtain best available screening tool for early assessment of delirium in hospitalized older people. There are so many delirium 
screening tools available and tested ranging from outdated till recent years. However, finding out more suitable screening tools that hopefully fit in the early assessment in delirium for hospitalized older people is the main reason of using systematic review to answer the PICO question.

The PICO question used in this study is "In hospitalized older people, how does early delirium assessment affect the outcome of care?" Keywords used to represent the population were "Hospitalized older people", "Hospitalized elderly", and "Hospitalized older adult" while keywords to represent the intervention were "Early delirium assessment" "Early delirium recognition" and "Early delirium prediction". At last, the keywords to represent the outcomes of care were "Mortality", "sedation medication", "Length of stay", and "Restrain". Searching limited to the year 1990 to 2016 and written in English in four databases which are CINAHL, OVID, PubMed, and Cochrane. The study selection process was preceded by identification of the title of the articles and or abstracts. Following by was selecting the eligible studies based on the inclusion and exclusion criteria.

The inclusion criteria were original studies related to providing care for older people who were hospitalized, study in older people (60 years old and over), study in the hospital-based service, Randomized Controlled Trial and non-Randomized Controlled Trial, written in English. The exclusion criteria in this study were study protocol, and study related to the community based intervention. Each abstract was reviewed by the authors to identify publications meeting the inclusion criteria. The full texts of the article were retrieved when the information in the abstract was insufficient. Data were collected where available on the age, sample size and English (or native language) speaking status of participants, number of patients with dementia, sensitivity, specificity, and the time interval between the delirium and the reference standard assessments.

After title screening, there were 133 articles found in total which included 97 articles from PubMed, 27 from CINAHL, and 9 from Cochrane Library. Screening for the abstract resulting 93 articles and finally, there are 4 articles met the inclusion and exclusion criteria of the study.

The following diagram shows the searching strategy and the result of the searching strategy conducted in this study: 


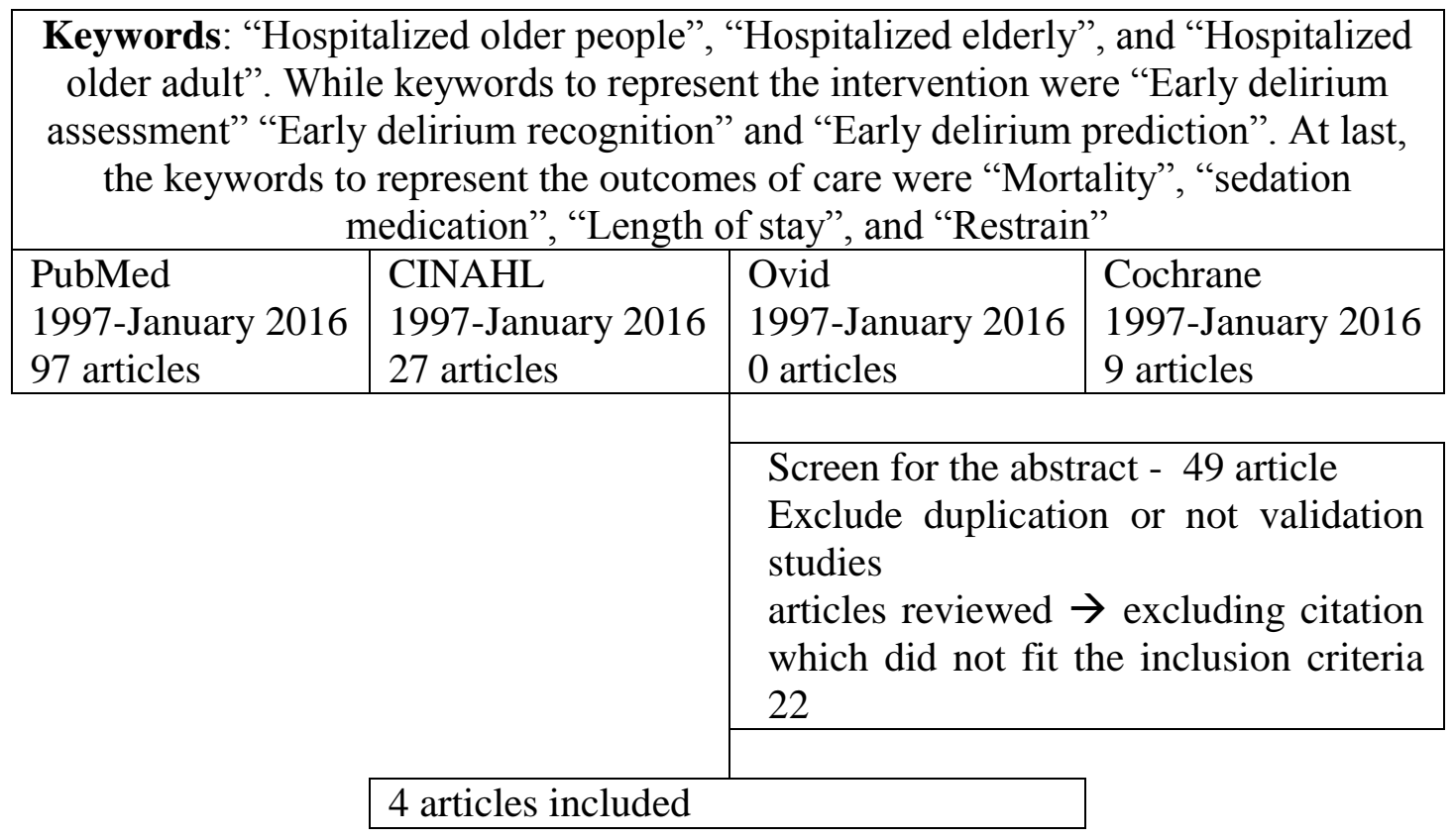

Diagram 1. Searching strategy flowchart applied in the study

\section{RESULTS}

\section{Delirium Screening Tools}

Delirium screening tool used in the studies was Confusion Assessment Method (CAM) (4/4 studies). Specifically, one of the study used NEECHAM to do the early screening before using CAM as follow up assessment. Another study used CAM integrated in comprehensive geriatric assessment (CGA) instead of performing CAM assessment itself. CAM used in different form, such as CAM-ICU (2/4 studies), and CAM-4 question (individually, or integrated in CGA). The screening tools were performed by both physician and or trained nurses. Nurses needed to perform NEECHAM screening first, so that, they were given training in using NEECHAM as a tool to screen delirium in their patients followed by CAM in following evaluation.

\section{Patient characteristics}

The most population age was older people, and all of the studies were conducted in European regions which are two studies from UK, one from Netherlands, and the last one is from Belgium. All studies were conducted specifically in the hospital based. In detail, 2 studies have similar settings which were in the ICU, and the rest was in the usual ward. The Mean age of all study was considered in the older age group, even the population criteria in certain study was adult age especially the study conducted in the ICU setting. 


\section{Identified early delirium assessment}

Assessment was done using CAM or CAM variation within 3 days after admission and during the hospitalization. CAM could be performed by either physician or nurses, but the training of using CAM was needed.

\section{Outcomes affected by early delirium assessment}

Outcomes measured in the articles were varied, each article gives the similar early assessment, which is using CAM or CAM variation. However, in the details, some of the articles looked for the effect in the use of haloperidol. While the rest were assessing length of stay, duration of delirium, and severity of the delirium. The use of haloperidol in early assessment of delirium may help patients to be treated with haloperidol $(9.9 \%$ to $14.8 \%, p<.00)$, with a lower dose (18 to $6 \mathrm{mg}, \mathrm{p}=.01$ ) and for a shorter time period ( 5 [IQR: 2-9] to 3 [IQR: 1-5] days, $p=.02$ ) (van den Boogaard et al., 2009). While the other study suggests that early assessment and use of haloperidol showed no change in delirium duration, but the use haloperidol seemed to reduce the need for sedatives.

Applying early delirium assessment integrated into the comprehensive geriatric assessment provided by the collaborative team showed improvement in the management of delirium, chronic pain, constipation, and urinary incontinence. Over twice as many patients were transferred to geriatric wards with mean days from admission to transfer fall from 10 to 3. Mean LOS fell by 4 days post-OPAL. Only the OPAL intervention was associated with LOS $(p=.02)$. Benchmarking data showed the LOS reduction to be greater than comparable hospitals in the UK (Harari, Martin, Buttery, O'Neill, \& Hopper, 2007). Using early delirium assessment lead by the nurses, duration of delirium was shorter - no more than 6 days, compared to the control group which was up to 12 days ( $p=.03)$ and the severity of delirium was less ( $p=.00)$ (Milisen et al., 2001).

\section{DISCUSSION}

\section{Early delirium assessment in hospitalized older people}

When it comes to the screening tools, there are so many delirium screening tools available and validated by the researchers around the world. One of the most validated, and used is the CAM (Thomas et al., 2012). Originally, CAM has nine items of questions to detect delirium in patients. In addition, through many researches and tailoring of the questionnaires, CAM is available in some new form by now (O'Regan et al., 2014). The new developed questionnaire stays the name of CAM as the core of the questionnaire is CAM. Some of the available CAMs are CAM (9 questions), CAM short form (4 questions), CAM-ICU, and CAM-FAM.

The high usability of CAM can be manifested as CAM is widely used in the world, as this questionnaire is already tested in many countries from around the world. Even CAM is already assessed in so many times, the sensitivity and specificity remain in a good shape (De \& Wand, 2015). Currently, one study reported that the sensitivity of CAM ranges from 40-100\%, while the specificity ranges from $63-100 \%$. The sensitivity decreases significantly on less trained operator, and training on using the questionnaire is needed (Wand et al., 2013). 
When it comes to assessing delirium in older people, CAM only takes several minutes, when compared to other questionnaire (De \& Wand, 2015). CAM is also available in some modified forms, which can be set up for different needs/settings. In addition, applying CAM also becomes more convenient in this day. CAM is available in some languages. Last, CAM is also widely used in an integrated way in comprehensive geriatric assessment, in which, when assessing comprehensive geriatric assessment, we also perform delirium screening in one time (Avelino-Silva et al., 2014).

\section{Outcomes of early delirium assessment in hospitalized older people}

There are so many papers about the validation of delirium screening tools that suggest the use of early delirium assessment or recognition in order to improve the prevention program, and early intervention to reduce the length of stay in the hospital. Outcomes that are suggested to be measured are delirium duration, severity of delirium, the use of sedation, length of stay, use of restraint, co-morbidity, and mortality. However, the papers on this study do not mention about the use of restraint, co-morbidity, and mortality. The researches included mostly prospective study but, there was no variable such as mortality instead of severity of delirium and length of stay.

Based on the result of the study, early assessment in delirium for hospitalized older people shows some advantages such as improving haloperidol use and reducing the length of stay in the hospital, delirium days, and severity of delirium (Harari et al., 2007; Milisen et al., 2001; Page et al., 2013; van den Boogaard et al., 2009).

Pro and cons of the use of haloperidol need further researches in order to provide better guidelines in providing the medication in suggesting ways. In the study in general, by using early delirium assessment, the use of haloperidol might increase (Page et al., 2013; van den Boogaard et al., 2009). In a lower dose and shorter time, it seems to reduce the need of sedatives, and promotes lower length of stay in hospital (Page et al., 2013).

Establishing the care team involving many disciplines will give a better way to improve the integrated care and collaborative care (Schraeder, Shelton, \& Sager, 2001) for older people which demonstrated in by Harari et al. (2007). It is not only to perform a better way to improve the care management, but also to improve the awareness in certain focus, such as delirium. In the care team, they can take part as the person who is responsible to do early assessment and later performs following assessment for the older people (Milisen et al., 2001). Furthermore, this care team which performs early delirium assessment may improve their delirium management program.

\section{Applying early delirium assessment in hospitalized older people in Indonesian setting}

There is a great urge to apply the early assessment in geriatric wards in Indonesia. There are some elements that Indonesia already has to apply early delirium assessment. First, geriatric wards have human resources (physician and nurses). Moreover, they can promote collaborative work in between the disciplines. Using CAM as early delirium assessment has been assessed to be effective in improving the outcome of care, and 
reduces mortality in advance. So that, applying the early delirium assessment can be one of a good thing that hospital can do to improve the quality of care (Harari et al., 2007).

Even if this early delirium assessment seems to be good to be applied in the clinical setting, geriatric wards in Indonesian hospital need to consider some other things such as the availability of CAM in Indonesian language, and the persons who have responsibility to perform CAM to the patients. Moreover, documentation is also needed on CAM. Before coming to the lack of training program, the available CAM or other screening tools remain questioned (Stukenberg et al., 2016). Currently, there is no strong evidence that CAM is really assessable, and stays reliable and valid in Indonesian language. Therefore, a need of further research in applying the early delirium assessment is high.

Considering both the strength and the weakness, applying this early assessment can be achieved in many ways. To overcome the translation process of the CAM, many research related to the validity and reliability of the tools showed high sensitivity and specificity. Later on, who will do the assessment actually there will be a collaborative way in performing delirium screening, which can be delivered by both physicians and nurses. According to Milisen et al. (2001), nurses can lead the delirium care for hospitalized older people by using collaboration of nurses to assess the early screening, physicians/psychiatrists diagnose the patient and prescribe the treatment, and nurses perform monitoring using similar delirium assessment.

The last part, to deal with the lack of training to perform the assessment, education center of the hospital together with their expertise might develop the appropriate training programs for either nurses or physicians to perform delirium assessment. So that, both disciplines work on the same way and a good collaboration can be promoted. Documentation might be still a big problem that even if those disciplines speak the similar language, but they do not have the media to build up this communication, then it is impossible to get through it well. Establishing the CAM assessment in the electronic medical record may seems to be far away from nowadays condition, but providing paper based assessment and specific page incorporating delirium care in older people can be a reasonable way to solve this problem.

\section{CONCLUSION}

Actually, there is no single study which concludes that early delirium assessment independently improves the outcomes. However, performing CAM integrated in comprehensive geriatric assessment can be the most important thing to be noted. Comprehensive geriatric assessment might seem to be over-assessed for hospitalized older people. Nevertheless, considering the advantages of CAM which are included in comprehensive geriatric assessment not only improve delirium prevent program but also prevent functional decline in advance. Applying early delirium assessment can shorten delirium time, reduce length of stay, and promote the appropriate use of sedation which affects the use of restrain indirectly, and predicts the mortality, and reduces mortality in advance.

Based on the literature, there might be a few papers related to assessing delirium screening or assessment tools in Indonesia, but this paper suggests conducting further 
research in providing delirium screening tool that is valid and reliable for Indonesian population. Later on, geriatric ward, or other wards in hospital to apply early delirium assessment in order to improve the care provided especially on delirium.

\section{REFERENCES}

Avelino-Silva, T. J., Farfel, J. M., Curiati, J. A. E., Amaral, J. R. G., Campora, F., \& Jacob-Filho, W. (2014). Comprehensive geriatric assessment predicts mortality and adverse outcomes in hospitalized older adults. BMC Geriatrics, 14, 129. doi:10.1186/1471-2318-14-129

Covinsky, K. E., Palmer, R. M., Fortinsky, R. H., Counsell, S. R., Stewart, A. L., Kresevic, D., Landefeld, C. S. (2003). Loss of independence in activities of daily living in older adults hospitalized with medical illnesses: increased vulnerability with age. Journal of the American Geriatrics Society, 51(4), 451-458. Retrieved from http://onlinelibrary.wiley.com/store/10.1046/j.15325415.2003.51152.x/asset/j.1532-

5415.2003.51152.x.pdf?v=1\&t=ijki1qva\&s=6865b27f6f437994b02f2783f432bd $29 \mathrm{e} 64 \mathrm{ce} 731$

De, J., \& Wand, A. P. (2015). Delirium Screening: A Systematic Review of Delirium Screening Tools in Hospitalized Patients. Gerontologist, 55(6), 1079-1099. doi:10.1093/geront/gnv100

Fagerberg, I., \& Engström, G. (2012). Care of the old-A matter of ethics, organization and relationships. International journal of qualitative studies on health and wellbeing, 7.

Gill, T. M., Allore, H. G., Holford, T. R., \& Guo, Z. (2004). Hospitalization, restricted activity, and the development of disability among older persons. Jama, 292(17), 2115-2124.

Retrieved

from http://jama.jamanetwork.com/data/Journals/JAMA/4950/JOC41058.pdf

Gillick, M. R., Serrell, N. A., \& Gillick, L. S. (1982). Adverse consequences of hospitalization in the elderly. Soc Sci Med, 16(10), 1033-1038.

Harari, D., Martin, F. C., Buttery, A., O'Neill, S., \& Hopper, A. (2007). The older persons' assessment and liaison team 'OPAL': evaluation of comprehensive geriatric assessment in acute medical inpatients. Age Ageing, 36(6), 670-675. doi:10.1093/ageing/afm089

Health, M. o. (2015). Data and Information: year 2014 (Indonesia Health Profile). Jakarta: Ministry of Health of Indonesia.

Hsieh, S. J., Ely, E. W., \& Gong, M. N. (2013). Can intensive care unit delirium be prevented and reduced? Lessons learned and future directions. Ann Am Thorac Soc, 10(6), 648-656. doi:10.1513/AnnalsATS.201307-232FR

Isfandiaty, R. H., Kuntjoro; Setiati, Siti; Roosheroe, Arya G. (2012). Incidence and Predictors for Delirium in Hospitalized Elderly Patients: a Restropective Cohort Study. Acta medica Indonesiana, 44(4), 290-297.

Milisen, K., Foreman, M. D., Abraham, I. L., De Geest, S., Godderis, J., Vandermeulen, E., Broos, P. L. O. (2001). A nurse-led interdisciplinary intervention program for delirium in elderly hip-fracture patients. Journal of the American Geriatrics Society, 49(5), 523-532 510p. doi:10.1046/j.1532-5415.2001.49109.x

MoH. (2014). Health Profile of Indonesia: Year 2013. Jakarta: Ministry of Health Republic of Indonesia. 
Nagamine, M., Jiang, H. J., \& Merrill, C. T. (2006). Trends in Elderly Hospitalizations, 1997-2004: Statistical Brief \#14 Healthcare Cost and Utilization Project (HCUP) Statistical Briefs. Rockville MD.

O'Regan, N. A., Ryan, D. J., Boland, E., Connolly, W., McGlade, C., Leonard, M., . . Timmons, S. (2014). Attention! A good bedside test for delirium? J Neurol Neurosurg Psychiatry, 85(10), 1122-1131. doi:10.1136/jnnp-2013-307053

Page, V. J., Ely, E. W., Gates, S., Zhao, X. B., Alce, T., Shintani, A., . . McAuley, D. F. (2013). Effect of intravenous haloperidol on the duration of delirium and coma in critically ill patients (Hope-ICU): a randomised, double-blind, placebocontrolled trial. Lancet Respir Med, 1(7), 515-523. doi:10.1016/s22132600(13)70166-8

Rigney, T. S. (2006). Delirium in the Hospitalized Elder and Recommendations for $\begin{array}{llll}\text { Practice. } & \text { Geriatric } & \text { Nursing, }\end{array}$ doi:http://dx.doi.org/10.1016/j.gerinurse.2006.03.014

Rosanti, E. (2006). Application of Professional Nursing Practice Model (Model Praktik Keperawatan Profesional) among nurses in medical ward. (Master), Gadjah Mada University, Universitas Gadjah Mada. (c.1 (0762-H-2006))

Schraeder, C., Shelton, P., \& Sager, M. (2001). The effects of a collaborative model of primary care on the mortality and hospital use of community-dwelling older adults. J Gerontol A Biol Sci Med Sci, 56(2), M106-112.

Schuurmans, M. J., Duursma, S. A., \& Shortridge-Baggett, L. M. (2001). Early recognition of delirium: review of the literature. Journal of Clinical Nursing, 10(6), 721-729. doi:10.1111/j.1365-2702.2001.00548.x

Stukenberg, S., Franck, M., Spies, C. D., Neuner, B., Myers, I., \& Radtke, F. M. (2016). How to advance prediction of postoperative delirium? A secondary analysis comparing three methods for very early assessment of elderly patients after surgery and early prediction of delirium. Minerva Anestesiol.

Tadd, W., Hillman, A., Calnan, S., Calnan, M., Bayer, T., \& Read, S. (2011). Dignity in Practice: An exploration of the care of older adults in acute NHS Trusts. NIHR SDO report.

Thomas, C., Kreisel, S. H., Oster, P., Driessen, M., Arolt, V., \& Inouye, S. K. (2012). Diagnosing Delirium in Older Hospitalized Adults with Dementia: Adapting the Confusion Assessment Method to International Classification of Diseases, Tenth Revision, Diagnostic Criteria. Journal of the American Geriatrics Society, 60(8), 1471-1477 1477p. doi:10.1111/j.1532-5415.2012.04066.x

van den Boogaard, M., Pickkers, P., van der Hoeven, H., Roodbol, G., van Achterberg, T., \& Schoonhoven, L. (2009). Implementation of a delirium assessment tool in the ICU can influence haloperidol use. Crit Care, 13(4), R131. doi:10.1186/cc7991

Wand, A. P., Thoo, W., Ting, V., Baker, J., Sciuriaga, H., \& Hunt, G. E. (2013). Identification and rates of delirium in elderly medical inpatients from diverse language groups. Geriatri Nurse, 34(5), 355-360. doi:10.1016/j.gerinurse.2013.05.004

World Population Ageing. (2013). New York: United nation. 